\title{
Shaping ability of Reciproc and TF Adaptive systems in severely curved canals of rapid microCT-based prototyping molar replicas
}

\author{
Ronald ORDINOLA-ZAPATA ${ }^{1}$, Clovis Monteiro BRAMANTE'1, Marco Antonio Hungaro DUARTE${ }^{1}$, Bruno Cavalini \\ CAVENAGO', David JARAMILLO², Marco Aurélio VERSIANI ${ }^{3}$
}

\footnotetext{
1- Department of Operative Dentistry, Endodontics and Dental Materials, Bauru School of Dentistry, University of São Paulo, Bauru, SP, Brazil.

2- Department of Endodontics, Loma Linda School of Dentistry, University of Loma Linda, Loma Linda, United States.

3- Department of Endodontics, Ribeirão Preto School of Dentistry, University of São Paulo, Ribeirão Preto, SP, Brazil.
}

Corresponding address: Ronald Ordinola-Zapata - Departamento de Dentística, Endodontia e Materiais Odontológicos - Faculdade de Odontologia de Bauru - USP - Al. Octávio Pinheiro Brisolla, 9-75 - 17012-901 - Bauru - São Paulo - Brazil - e-mail: ronaldordinola@usp.br

Submitted: December 19, 2013 - Modification: February 19, 2014 - Accepted: February 21, 2014

\begin{abstract}
$\mathrm{O}$ bjective: To evaluate the shaping ability of Reciproc and Twisted-File Adaptive systems in rapid prototyping replicas. Material and Methods: Two mandibular molars showing $\mathrm{S}$-shaped and 62-degree curvatures in the mesial root were scanned by using a microcomputed tomography $(\mu \mathrm{CT})$ system. The data were exported in the stereolitograhic format and 20 samples of each molar were printed at $16 \mu \mathrm{m}$ resolution. The mesial canals of 10 replicas of each specimen were prepared with each system. Transportation was measured by overlapping radiographs taken before and after preparation and resin thickness after instrumentation was measured by $\mu C T$. Results: Both systems maintained the original shape of the apical third in both anatomies $(P>0.05)$. Overall, considering the resin thickness in the 62-degree replicas, no statistical difference was found between the systems $(P>0.05)$. In the S-shaped curvature replica, Reciproc significantly decreased the thickness of the resin walls in comparison with TF Adaptive. Conclusions: The evaluated systems were able to maintain the original shape at the apical third of severely curved mesial canals of molar replicas.
\end{abstract}

Keywords: Dental pulp cavity. X-ray microtomography. Anatomy. Molar.

\section{NTRODUCTI ON}

Preparation of root canal system includes both enlargement and shaping of the complex endodontic space together with its disinfection ${ }^{10}$. To fulfill these requirements, a variety of manual, rotary, and reciprocating instruments have been developed. In the literature, several models have been proposed for the laboratory assessment of post-operative root canal preparation; however, investigations have been usually performed with extracted teeth. The major advantage of using teeth is the reproduction of the clinical situation, but the wide range of variations in the three-dimensional root canal morphology makes the standardization of the sample difficult ${ }^{24}$. Consequently, the results might demonstrate the effect of canal anatomy rather than the variable of interest ${ }^{7}$. Besides, bioethical concerns and the potential for cross-infection originated from contaminated specimens are a current threat to such practice in some institutions.

Considering that the main role of laboratorybased studies is to develop well-controlled conditions that allow to reliably compare certain factors, Weine, et al. ${ }^{26}$ (1975) formerly proposed the use of simulated root canals in clear casting resin, which could be created to any predetermined diameter, shape, or curvature, for the assessment of root canal preparation. This model guarantees a high degree of reproducibility and standardization of the experimental design ${ }^{10}$ and has been used in several investigations ${ }^{10}$. Nevertheless, plastic blocks present some disadvantages such as absence of multiplanar curvatures ${ }^{5}$, anatomical irregularities ${ }^{25}$, or convexities, generally presented in teeth.

A new set of manufacturing technologies has 
been introduced in the past decade to offer support for research tasks that require prototypes. These new techniques are typically referred to as rapid prototyping technologies, and they allow prototypes to be produced in a wide range of materials with notable precision ${ }^{8,12}$. These recent technologies offer an optimized precision of a few micrometers or even hundreds of nanometers ${ }^{8}$. Because of this precision, these technologies are specifically applicable to biomedical engineering ${ }^{12}$. In Dentistry, high definition models in resin can be produced using 3D printers, on automatic, additive, layerby-layer manufacturing processes, with resolution ranging from 16 to $32 \mu \mathrm{m}$ per layer, from obtained computer-aided design (CAD) models of the object in standard tessellation language (.stl) format, generated from micro-computed tomography (micro-CT) scanning.

The reproduction of natural teeth in transparent rapid prototyping replicas is very promising and has the potential to be included in the specialty for educational purposes and endodontic training ${ }^{14}$. In addition, it allows for a complete standardization of the sample when evaluating the root canal instrumentation in laboratory-based studies. To date, literature lacks studies using rapid prototyping tooth replicas on post-operative canal preparation with different instruments and techniques. Thus, the aim of this study was to evaluate the shaping ability of Reciproc and Twisted-File Adaptive systems in curved mesial canals of rapid prototyping mandibular molar replicas, using three-dimensional printing technology based on micro-CT image data.

\section{MATERIAL AND METHODS}

Digital data of two extracted human mandibular molars with fully formed apices presenting separated roots and showing curvatures at varying degrees in the mesial root were selected from a microcomputed tomography image bank. Digital data of the teeth were selected on the basis of the curvature angle, as previously described ${ }^{9,17}$. One of the specimens presented a continuous 62-degree curvature of the mesial root (Figure $1 \mathrm{~A}$ ), while the other had an S-shaped mesial root with 21 and 32 degrees in the primary (apical level) and secondary (middle level) curvatures, respectively (Figure 1B).

\section{Micro-CT scanning}

Originally, each tooth was slightly dried, mounted on a custom attachment, and scanned in a micro-CT scanner (SkyScan 1174v2; BrukermicroCT, Kontich, Belgium) at an isotropic resolution of $18 \mu \mathrm{m}$. The $\mathrm{X}$-ray tube was operated at $50 \mathrm{kV}$ and $800 \mathrm{~mA}$, and the scanning was performed by $180^{\circ}$ rotation around the vertical axis with a rotation step of 1.0 , using a $1.0-\mathrm{mm}$ thick aluminum filter. Images of each specimen were reconstructed with dedicated software (NRecon v.1.6.3; Bruker-microCT, Kontich,



Figure 1- Mandibular molar specimens showing a continuous 62-degree curvature of the mesial root (A) and a S-shaped mesial root (B). Respective replicas are also presented (C - D). Microcomputed tomography transversal sections show a Vertucci Type I configuration in both mesial roots with a ribbon-shaped canal from the cervical to the apical third. A decrease of the radiolucent area in the root canal space of molar replicas is related to the supporting material used during the printing process (wax). The reproduction of accessory anatomy as isthmuses and lateral canals can be observed in respective microcomputed tomography sections of molar replicas 
Belgium), providing axial cross sections of the inner structure of the samples. Three-dimensional models of the teeth in a stereolitographic format (.stl) adjusted to 600,000 triangles resolution, from the apex to $1 \mathrm{~mm}$ above the pulp chamber level, were generated by the binarization process using CTAn v.1.12 software (Brucker-microCT, Kontich, Belgium). An analysis of the internal anatomy of the teeth revealed a Vertucci Type I configuration in both mesial roots with a ribbon-shaped canal at the cervical and middle third of the root (Figures $1 \mathrm{~A}$ and $1 \mathrm{~B})$.

\section{D-printing of prototyping molar replicas}

The three-dimensional models of each tooth, in .stl format, were exported to a Projet HD3500 3D-printer machine (3D system, Rock Hill, SC, USA), which laid down successive layers of clear resin at $16 \mu \mathrm{m}$ resolution (Visijet Crystal, 3D system, Rock Hill, SC, USA) to build a real replica of the specimens (Figures $1 \mathrm{C}$ and 1D). Twenty prototypes of each tooth were printed.

\section{I nitial sample preparation}

Canal preparations were carried out in the mesial root of the replicas. The operator was an endodontist with expertise in rotary and reciprocating techniques, and after a training period using resin-based prototyping molars. After an initial irrigation with $1 \% \mathrm{NaOCl}$, a \#10 K-file was introduced into the mesial canal system using a balanced force technique until it reached the apical foramen. Then, buccal-lingual digital radiographic (Gnatus XR6010, Ribeirão Preto, SP, Brazil) images were taken for each prototype using the parallel technique and a custom device (Krystal-X easy, Owandy RadioVision, Gragny, France) so that the tooth could be placed in the same position before and after preparation, as previously described ${ }^{2}$. All digital images were captured and stored.

\section{Canal preparation and irrigation}

Glide path was achieved with PathFile 1, 2, and 3 (Dentsply Maillefer, Ballaigues, Switzerland) $1.0 \mathrm{~mm}$ from the apical foramen. Then, the molar replicas of each specimen $(n=20)$ were randomly distributed into 2 groups $(n=10)$, according to the instrumentation technique system used to prepare the mesial canal: Reciproc single file technique and Twisted-File Adaptive (TF Adaptive).

In the Reciproc technique, the R25 (VDW GmbH, Munich, Germany) instrument was introduced into the canal until resistance was felt and then activated in reciprocating motion generated by a $6: 1$ contraangle hand-piece (Sirona, Bensheim, Germany) powered by an electric motor (VDW Silver; VDW GmbH, Munich, Germany). TF Adaptive size 25.06 followed by a 30.06 instrument were taken carefully into the canal until the working length was achieved, using a Sybron Elements motor (SybronEndo, Glendora, CA, USA) selected in the adaptive motion. Each instrument in the Reciproc and TF adaptive technique was moved in apical direction using an in-and-out pecking motion of about $3 \mathrm{~mm}$ in amplitude with a light apical pressure. After three pecking motions, the instrument was removed from the canal and cleaned.

Copious irrigation with $1 \% \mathrm{NaOCl}$ was performed during root canal instrumentation and a final rinse with $10 \mathrm{~mL}$ of $70 \%$ alcohol was used to eliminate resin debris, in order to improve the transparency of the replicas, using a syringe with a 29-gauge needle (Endo Eze; Ultradent Products Inc., South Jordan, UT, USA). For each experimental group, five sets of instruments were used.

\section{Evaluation of canal transportation}

After shaping procedure, a standardized digital radiograph of the mesial canal was obtained with the final instrument positioned at the working length, following the aforementioned parameters. The digital images taken before and after the canal preparation were overlapped by using Adobe Photoshop software (Adobe Systems, San Jose, CA, USA), and Image Tool software (UTHSC, San Antonio, Texas, USA) was used to measure the angle of the curvature before and after instrumentation (Figure 2A). The difference between them was considered as the amount of transportation ${ }^{1}$. In the replicas presenting an S-shaped configuration of the mesial root, angles of either primary (apical level) or secondary (middle third) curvatures were measured (Figure 2B).

\section{Evaluation of remaining resin thickness}

The prepared prototype molars were also submitted to a micro-CT scanning and reconstruction following the aforementioned parameters. Using Dataviewer v.1.4.4 software (Bruker-microCT, Kontich, Belgium), the replicas were properly aligned and the minimal remaining resin thickness, in the distal and mesial walls of the instrumented root canals, at $1-\mathrm{mm}$ interval from the working length to the furcation level of the mesial root, was measured in $\mathrm{mm}$ by using the Dataviewer software (Bruker-microCT, Kontich, Belgium). Considering the size of the mesial root in each type of replica, it was possible to evaluate $10 \mathrm{~mm}$ of the root in the 62-degree curvature prototypes and $8 \mathrm{~mm}$ in the S-shaped curvature prototypes.

\section{Statistical analysis}

Considering that the data of canal transportation and remaining resin thickness were normally distributed (Shapiro-Wilk test; P>0.05), they were presented as means and standard deviations and 


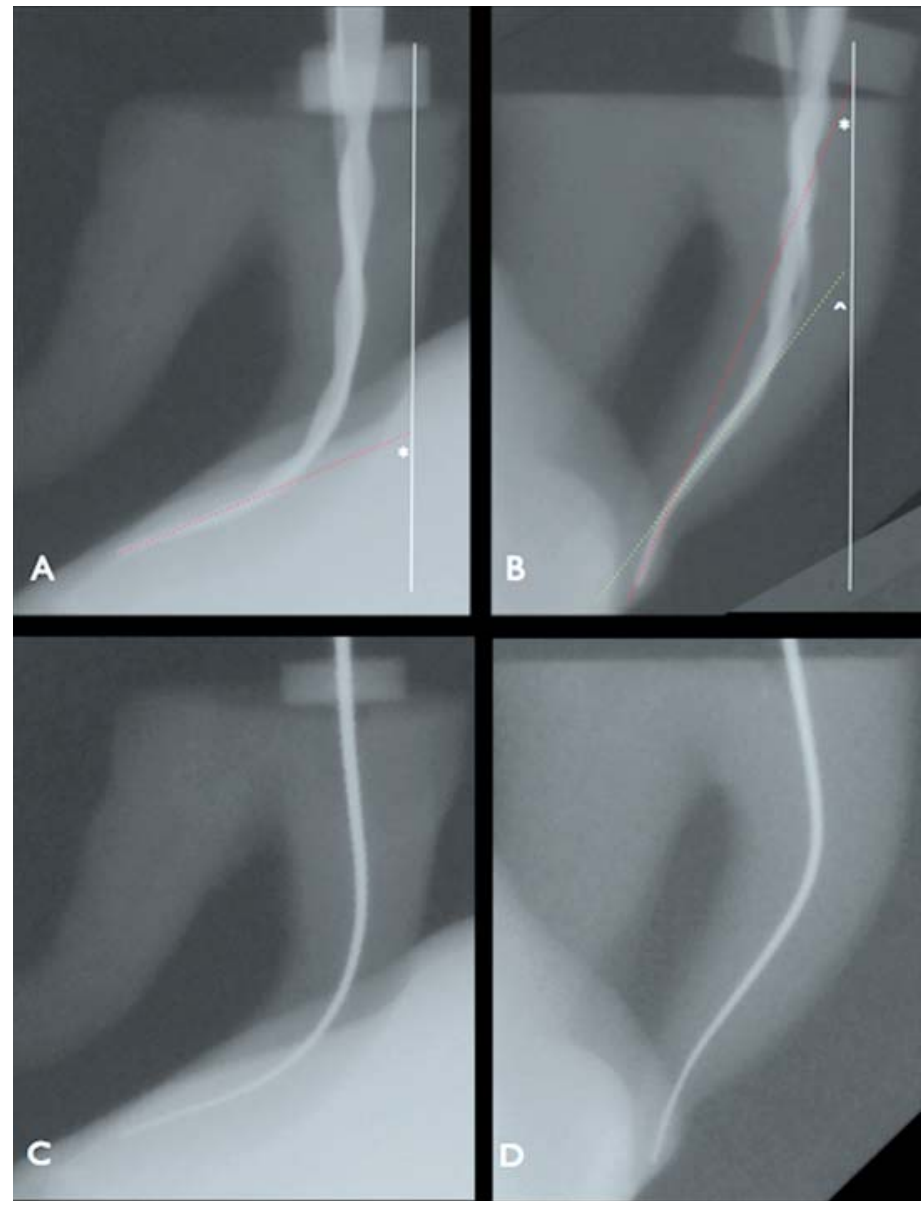

Figure 2- Radiographic images after superimposition of 62-degree (A) and "S"-shaped (B) root canal curvatures of molar replicas. The angle $\left(^{*}\right)$ was formed by the intersection of the root canal axis at the apical third (red line) and the long axis that is perpendicular to the coronal level (white line). The evaluation of the S-shaped curvature included the measurement of the angles of the primary $\left(^{*}\right)$ and of the secondary curvature $\left(^{\wedge}\right)$. Preoperative radiographs are shown in (C-D)

statistically compared using unpaired Student's t-test. Statistical analysis was performed using Graphpad Prisma (SPSS Inc., Chicago, IL, USA) with a significance level set at $5 \%$.

\section{RESULTS}

No ledge, fracture, perforation or stripping were observed in all samples.

\section{Canal transportation}

The digital overlapped radiographs taken before and after the canal preparation showed that both systems maintained the original shape of the curved canals in both types of replicas, especially at the apical third (Figure 2). No statistical difference was observed between Reciproc and TF Adaptive systems in the degree of transportation in the replicas with 62 -degree of curvature $(P>0.05)$, which was less than $1^{\circ}$ in all samples. In the primary curvature (apical level) of the S-shaped replicas, Reciproc and TF Adaptive systems showed a mean degree of transportation of $2.3^{\circ} \pm 0.7$ and $1.6^{\circ} \pm 0.9$, respectively, with no statistical difference between them $(P>0.05)$. On the other hand, the secondary curvature (middle level) of the S-shaped replicas showed a significantly higher transportation after using Reciproc instrument $\left(18.4^{\circ} \pm 1.2\right)$ than TF Adaptive system $\left(1.3^{\circ} \pm 1.2\right)(P<0.05)$.

\section{Remaining resin thickness}

Table 1 shows the mean ( \pm standard deviation) of the remaining resin thickness at the mesial and distal walls of the mesial root of the prototypes after canal preparation with Reciproc and TF Adaptive systems.

Considering the 62-degree replicas, no statistical difference was found between the systems in the mesial and distal walls in all levels $(P>0.05)$, except in the 2 and $3 \mathrm{~mm}$ from the apex, in which Reciproc showed significantly less remaining resin thickness than TF Adaptive $(P<.05)$. In the $S$-shaped curvature molar replica, no difference was observed between the systems in the furcation level (level 8) in both mesial and distal walls ( $P>0.05)$. However, overall, Reciproc significantly decrease the thickness of resin walls in comparison with TF Adaptive $(\mathrm{P}<0.05)$. 
Table 1- Mean and standard deviation of the amount of resin thickness at the mesial and distal walls of the mesial root of both prototyping molar replicas, in different levels, after root canal preparation with Reciproc and Twisted-File Adaptive systems

\begin{tabular}{|c|c|c|c|c|c|c|c|}
\hline & \multirow[b]{2}{*}{$\begin{array}{l}\text { Root Levels } \\
\text { From Apex } \\
\text { (in mm) }\end{array}$} & \multicolumn{2}{|c|}{ S-shaped curvature } & \multirow[b]{2}{*}{$P$} & \multicolumn{2}{|c|}{ 63-degree curvature } & \multirow[b]{2}{*}{$P$} \\
\hline & & Reciproc & Twisted-File & & Reciproc & Twisted-File & \\
\hline \multirow[t]{10}{*}{ Mesial Wall } & 1 & $0.28 \pm 0.05$ & $0.42 \pm 0.05$ & * & $0.77 \pm 0.07$ & $0.87 \pm 0.13$ & ns \\
\hline & 2 & $0.40 \pm 0.06$ & $0.67 \pm 0.12$ & * & $0.86 \pm 0.05$ & $1.04 \pm 0.04$ & * \\
\hline & 3 & $0.89 \pm 0.08$ & $1.08 \pm 0.07$ & * & $0.84 \pm 0.10$ & $0.97 \pm 0.08$ & * \\
\hline & 4 & $1.17 \pm 0.08$ & $1.18 \pm 0.08$ & $\mathrm{~ns}$ & $0.89 \pm 0.06$ & $0.90 \pm 0.06$ & ns \\
\hline & 5 & $1.34 \pm 0.08$ & $1.24 \pm 0.08$ & * & $1.07 \pm 0.07$ & $1.08 \pm 0.16$ & ns \\
\hline & 6 & $1.58 \pm 0.05$ & $1.34 \pm 0.06$ & * & $1.04 \pm 0.04$ & $1.07 \pm 0.06$ & ns \\
\hline & 7 & $1.61 \pm 0.04$ & $1.52 \pm 0.06$ & ns & $1.12 \pm 0.05$ & $1.16 \pm 0.07$ & ns \\
\hline & 8 & $1.66 \pm 0.02$ & $1.64 \pm 0.07$ & ns & $1.22 \pm 0.06$ & $1.26 \pm 0.07$ & ns \\
\hline & 9 & - & - & - & $1.38 \pm 0.16$ & $1.44 \pm 0.11$ & ns \\
\hline & 10 & - & - & - & $1.62 \pm 0.09$ & $1.66 \pm 0.07$ & ns \\
\hline \multirow[t]{10}{*}{ Distal Wall } & 1 & $0.46 \pm 0.08$ & $0.48 \pm 0.03$ & ns & $0.66 \pm 0.05$ & $0.61 \pm 0.08$ & ns \\
\hline & 2 & $0.77 \pm 0.07$ & $0.68 \pm 0.03$ & * & $0.69 \pm 0.05$ & $0.68 \pm 0.08$ & ns \\
\hline & 3 & $0.88 \pm 0.05$ & $0.83 \pm 0.06$ & ns & $0.76 \pm 0.09$ & $0.72 \pm 0.05$ & ns \\
\hline & 4 & $0.71 \pm 0.08$ & $0.98 \pm 0.10$ & * & $0.86 \pm 0.06$ & $0.85 \pm 0.09$ & ns \\
\hline & 5 & $0.60 \pm 0.06$ & $1.00 \pm 0.10$ & * & $0.93 \pm 0.21$ & $0.96 \pm 0.16$ & ns \\
\hline & 6 & $0.43 \pm 0.05$ & $0.87 \pm 0.09$ & * & $0.72 \pm 0.17$ & $0.73 \pm 0.07$ & ns \\
\hline & 7 & $0.47 \pm 0.04$ & $0.73 \pm 0.09$ & * & $0.59 \pm 0.13$ & $0.61 \pm 0.15$ & ns \\
\hline & 8 & $0.93 \pm 0.08$ & $0.88 \pm 0.10$ & ns & $0.78 \pm 0.08$ & $0.74 \pm 0.11$ & ns \\
\hline & 9 & - & - & - & $1.04 \pm 0.12$ & $1.03 \pm 0.11$ & ns \\
\hline & 10 & - & - & - & $1.44 \pm 0.12$ & $1.40 \pm 0.13$ & ns \\
\hline
\end{tabular}

* Statistically significant difference $(P<0.05)$; ns: not significant

\section{DISCUSSION}

Dental students can improve their dental handskill training from several sources. Usually they practice on extracted teeth or in patients under supervision of dental experts. However, some complications can be found during the training such as: the difficulty in finding human teeth for preclinical training and the unavailability of real challenging cases $^{16}$.

With recent advances in rapid prototyping technology, the fabrication of real three-dimensional models of human organs for medical and dental surgery has been introduced ${ }^{12,13}$. Rapid prototyping is an expression that represents a technology based on the construction of physical three-dimensional structures based on its respective virtual models ${ }^{4}$, and have been used in dental therapy, mainly for surgical planning in implantology and maxillofacial prostheses ${ }^{19}$. Micro-CT is a nondestructive technique that can be used for obtaining digital information on the 3-D geometries of solid objects and from which structural parameters can be derived (.stl file) to produce rapid prototype models, as in the present study. The STL file contains a description of the boundary surfaces of the model that is sufficient to use as input to a rapid prototyping system through layer-by-layer polymerization of a photosensitive resin ${ }^{3}$.

Despite the aforementioned advantages, in endodontics, resin-made prototypes have a critical limitation considering the obvious difference between the hardness of dentin and resin. In previous reports, the major drawbacks of using rotary instruments in resin blocks were the heat generated, which may soften the resin material, and instrument separation, because of binding of its cutting blades ${ }^{11,18}$. In the present study, none of these drawbacks was observed, probably due to differences in composition between resin blocks and resin-made prototypes. The first step towards increasing the level of patient safety in endodontic treatment is for all clinicians to acquire knowledge and skills in the early stage of training. Endodontic 
treatment, like other disciplines of Dentistry, can be associated with unwanted or unforeseen procedural errors $^{21}$. In this way, preclinical endodontic skill training with rapid prototyping teeth provides a new opportunity that is difficult to realize otherwise: to perform endodontic treatment with realistic three-dimensional models of teeth presenting any type of canal configuration that exists in natural teeth. At some point in the preclinical training, different configurations of the root canal may be presented to the students by adding progressive difficulty. Another advantage of these prototypes is that the root canal complexities can be replicated several times allowing clinicians to practice the procedures as many times as they want, using different protocols. Similarly, in the research field, standardization of the three-dimensional root canal morphology of the sample is an important issue ${ }^{24}$. Due to the accuracy of the printing procedure ( $0.025 \mathrm{~mm}$ per inch), it is possible to compare experimental groups under similar anatomical condition.

In the present study, rapid prototyping models of mandibular molars with complex curvatures of the mesial root were used to evaluate two recently launched preparation systems: Reciproc and TF Adaptive. The Reciproc instrument has been designed specifically for use in reciprocation instead of the conventional continuous rotation method ${ }^{23}$. The reciprocating movement aims to minimize the risk of instrument fracture caused by torsional stress as the angle of counterclockwise rotation (cutting direction) was designed to be smaller than the elastic limit of the instrument ${ }^{15}$. On the other hand, the TF Adaptive System, when used with the Elements Motor with Adaptive Motion Technology, rotates clockwise and, depending on the load on the file, adapts and reverses counter-clockwise by coasting in a reciprocating motion ${ }^{22}$.

The present results showed that the straightening of the canal at the apical third was similar for both instruments, which corroborate previous findings using human teeth ${ }^{2,6,20}$. It was also observed that the lesser amount of resin thickness after canal instrumentation was located at the distal wall of cervical third. According to Stern, et al. ${ }^{20}$ (2012), root canal shaping with rotary nickel-titanium instruments tends to move the preparation towards the furcal aspect of the root at the cervical third, probably because of the increase volume of the canal up to three times at this point ${ }^{20}$.

The differences observed between the instruments regarding resin thickness at the coronal level in S-shaped anatomy and at the apical third of 62-degree prototype may be related to the larger metal mass of Reciproc compared with TF Adaptive. In the S-shaped curvature prototype, the tendency of Reciproc to leave less dentin in either mesial or distal walls may be associated with the physical properties of resin, which is softer than dentin, and the larger taper and stiffness of Reciproc in comparison with TF Adaptive. Despite this, both systems respected original root canal curvature well, especially at the apical level, and caused no iatrogenic events such as zipping, strip perforations or ledging.

Whereas this study aimed at studying the shaping ability of two endodontic systems in rapid-prototyping resin teeth, further studies of the behavior of these systems in teeth with different anatomical configurations still need to be conducted. In addition, the use of microCTbased rapid prototyping resin teeth is promising for educational purposes, endodontic training and research.

\section{CONCLUSION}

The present data suggest that microCT-based rapid prototyping resin teeth may be a valuable adjunct to endodontic training. Reciproc and TF Adaptive systems were able to maintain the original shape at the apical third of severely curved mesial canals of molar replicas.

\section{ACKNOWLEDGEMENTS}

The authors deny any conflicts of interest. Supported by the Brazilian Funding Agency FAPESP - São Paulo Research Foundation grants 2013/03695-0 2009/09989-0, 2010/16002-4, and 2010/16072-2.

\section{REFERENCES}

1- Burklein S, Benten S, Schäfer E. Shaping ability of different single-file systems in severely curved root canals of extracted teeth. Int Endod J. 2013;46:590-7.

2- Burklein S, Hinschitza K, Dammaschke T, Schäfer E. Shaping ability and cleaning effectiveness of two single-file systems in severely curved root canals of extracted teeth: Reciproc and WaveOne versus Mtwo and ProTaper. Int Endod J. 2012;45:449-61. 3. Chan DC, Frazier KB, Tse LA, Rosen DW. Application of rapid prototyping to operative dentistry curriculum. J Dent Educ. 2004;68:64-70.

4- Chua CK, Leong KF, Lim CS. Rapid prototyping: principles and applications. $3^{\text {rd }}$. ed. Singapore: World Scientific Publishing; 2010. 5- Cunningham CJ, Senia ES. A three-dimensional study of canal curvatures in the mesial roots of mandibular molars. J Endod. 1992;18:294-300.

6- Çelik D, Taşdemir T, Er K. Comparative study of 6 rotary nickel-titanium systems and hand instrumentation for root canal preparation in severely curved root canals of extracted teeth. J Endod. 2013;39:278-82.

7- De-Deus G. Research that matters - root canal filling and leakage studies. Int Endod J. 2012;45:1063-4.

8- Derby B. Printing and prototyping of tissues and scaffolds. Science. 2012;338:921-6.

9- Hankins P, EIDeeb M. An evaluation of the Canal Master, balanced-force, and step-back techniques. J Endod. 1996;22:12330 . 
10- Hulsmann M, Peters OA, Dummer PM. Mechanical preparation of root canals: shaping goals, techniques and means. Endod Topics. 2005;10:30-76.

11- Kum KY, Spängberg L, Cha BY, Il-Young J, Seung-Jong L, ChanYoung L. Shaping ability of three ProFile rotary instrumentation techniques in simulated resin root canals. J Endod. 2000;26:71923.

12- Lantada AD, Morgado PL. Rapid prototyping for biomedical engineering: current capabilities and challenges. Annu Rev Biomed Eng. 2012;14:73-96.

13- Lee SJ, Jung IY, Lee CY, Choi SY, Kum KY. Clinical application of computer-aided rapid prototyping for tooth transplantation. Dent Traumatol. 2001;17:114-9.

14- Michmershuizen F. Redefining endodontic education: L. Stephen Buchanan discusses new ways to help dentists learn. Roots. 2012;3:30-2.

15- Plotino G, Grande NM, Testarelli L, Gambarini G. Cyclic fatigue of Reciproc and WaveOne reciprocating instruments. Int Endod J. 2012;45:614-8.

16- Rhienmora P, Haddawy P, Khanal P, Suebnukarn S, Dailey MN. A virtual reality simulator for teaching and evaluating dental procedures. Methods Inf Med. 2010;49:396-405.

17- Schäfer E, Diez C, Hoppe W, Tepel J. Roentgenographic investigation of frequency and degree of canal curvatures in human permanent teeth. J Endod. 2002;28:211-6.

18- Schäfer E, Erler M, Dammaschke T. Influence of different types of automated devices on the shaping ability of rotary nickeltitanium FlexMaster instruments. Int Endod J. 2005;38:627-36.
19- Soares PV, Almeida Milito G, Pereira FA, Reis BR, Soares CJ, Sousa Menezes M, et al. Rapid prototyping and 3D-virtual models for operative dentistry education in Brazil. J Dent Educ. 2013;77:358-63.

20- Stern S, Patel S, Foschi F, Sherriff M, Mannocci F. Changes in centring and shaping ability using three nickel-titanium instrumentation techniques analysed by micro-computed tomography. Int Endod J. 2012;45:514-23.

21- Suebnukarn S, Haddawy P, Rhienmora P, Gajananan K. Haptic virtual reality for skill acquisition in endodontics. J Endod. 2010;36:53-5.

22- Twisted file adaptive system. [website]. 2013 [cited Feb. 19, 2014]. Available from: http://axis.sybronendo.com/tfadaptive_ confidence.

23- Versiani MA, Leoni GB, Steier L, De-Deus G, Tassani S, Pécora JD, et al. Micro-computed tomography study of oval-shaped canals prepared with the self-adjusting file, Reciproc, WaveOne, and ProTaper universal systems. J Endod. 2013;39:1060-6.

24- Versiani MA, Pécora JD, Sousa-Neto MD. Microcomputed tomography analysis of the root canal morphology of single-rooted mandibular canines. Int Endod J. 2013;46:800-7.

25- Villas-Bôas MH, Bernardineli N, Cavenago BC, Marciano M, Del Carpio-Perochena A, Moraes IG, et al. Micro-computed tomography study of the internal anatomy of mesial root canals of mandibular molars. J Endod. 2011;37:1682-6.

26- Weine FS, Kelly RF, Lio PJ. The effect of preparation procedures on original canal shape and on apical foramen shape. J Endod. $1975 ; 1: 255-62$ 